\title{
“¡OYE, TÚ, BATO!, ¿DÓNDE ESTÁN LOS PLEBES Y TU MORRA?” APROXIMACIÓN LEXICOLÓGICA, MEDIANTE LA DIALECTOLOGÍA PERCEPTUAL, AL HABLA DE BAHÍA DE KINO Y GUAYMAS, SONORA.
}

\author{
";Oye, tú, bato!, ¿Where are the plebes and your morra? Lexicological approach, thorough the \\ Perceptual Dialectology, to the Bay of Kino and Guaymas, Sonora
}

Aline Granados*

\begin{abstract}
RESUMEN
El presente artículo tiene como propósito estudiar la variación léxica en dos puertos pesqueros del estado de Sonora: Bahía de Kino y Guaymas. El marco teórico a seguir será el de la Lingüística Folk (Niedzielski \& Preston, 2003), Dialectología Perceptual (Gould \& White, 1972) y Dennis Preston (1989). Se estudiarán las palabras morro, plebe y bato así como sus diferentes usos entre la población de ambos puertos. De esta forma, en este trabajo se concluye que ambos puertos son, por los menos a nivel léxico, dos comunidades de habla distintas respecto al vocablo bato (Bloomfield, 1933).

Palabras clave: Dialectología perceptual, lingüística folclórica, variación léxica, Kino bay, Guaymas.
\end{abstract}

\section{ABSTRACT}

In this paper I will study lexical variation in two fisherman's towns in Sonora state: Kino bay and Guaymas. The theoretical framework for the present study lies on Folk Linguistics (Niedzielski \& Preston, 2003), Perceptual Dialectology (Gould \& White, 1972) and Dennis Preston (1989). In this paper I will study words as morro, plebe and bato and its different uses among the population of both towns. Finally, I conclude that Kino Bay and Guaymas are two different speech communities (Bloomfield, 1933) at least, in the use of the noun bato.

Key Words: Perceptual Dialectology, Folk Linguistics, Lexical variation, Kino bay, Guaymas. 


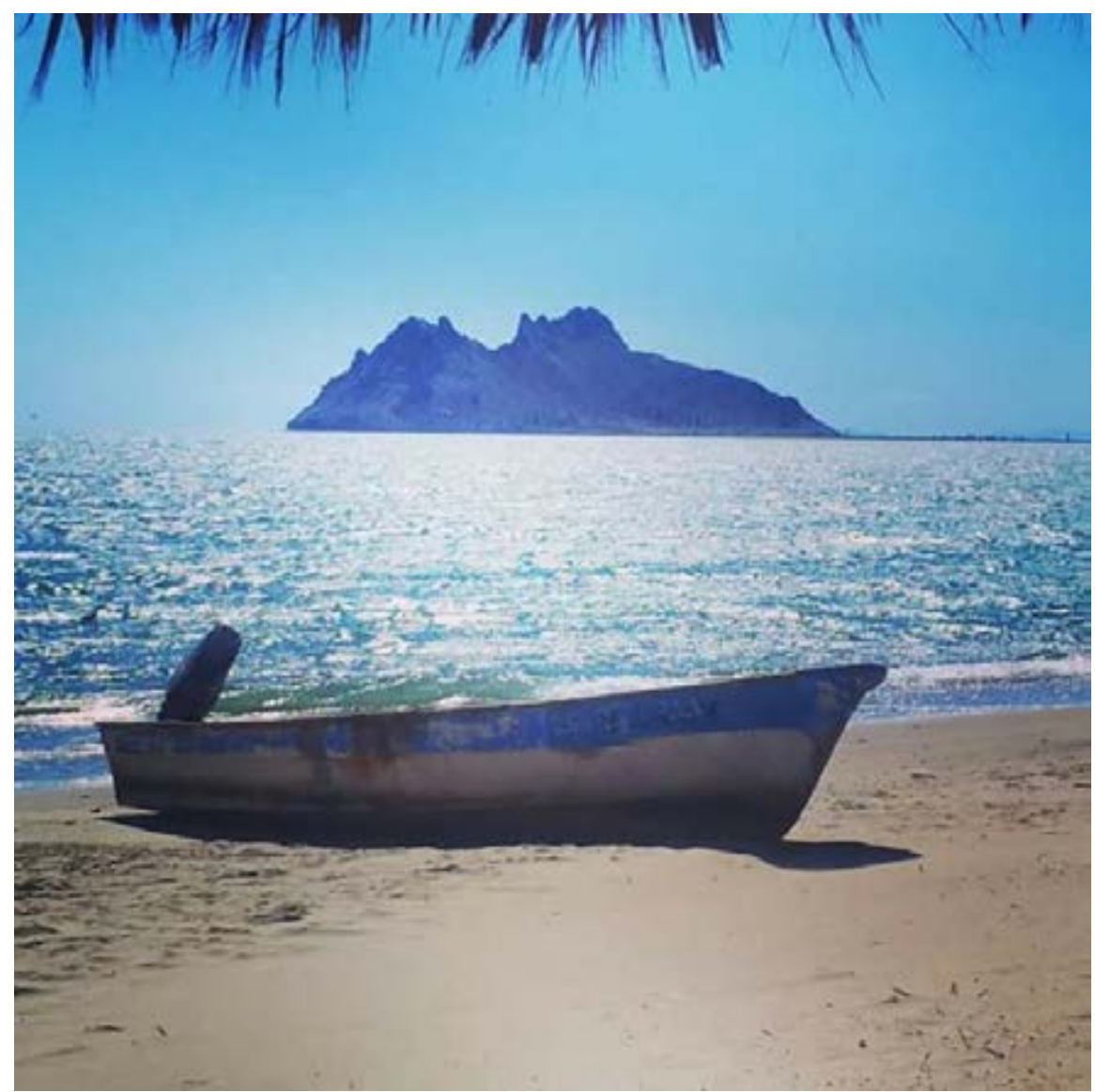

FIGURA 1. Isla Alcatraz ${ }^{1}$

\section{Introducción}

La región costera sonorense presenta características fónicas y léxicas muy interesantes. Este trabajo centrará su atención en estudiar tres palabras emblemáticas de la región: morro, bato y plebe. El marco interpretativo del presente trabajo será el de la lingüistica folk (Niedzielski \& Preston, 2003). En el primer apartado se hará un somero recorrido por los principales conceptos y antecedentes de dicho marco conceptual, así como de los estudios previos para después analizar cada palabra por separado. La base de observación sobre el uso y significado de tales términos la constituyen las respuestas a un cuestionario recogido con
24 informantes entrevistados en el año 2013 durante una estancia de dos semanas, en el puerto de Bahía de Kino, y dos semanas en el puerto de Guaymas, Sonora.

\section{Lingüística folk}

En el presente trabajo se seguirán los postulados de la lingüística folk, cuyos pioneros son Niedzielski y Preston (2003). Esta corriente centra su atención en las reacciones conscientes e inconscientes que manifiestan los hablantes ante distintos hechos del lenguaje, así como los juicios subjetivos que estos emiten ${ }^{2}$. En opinión de Preston (1989), las creencias de los 
hablantes legos (o folk concepts) forman parte de la cultura de una comunidad de habla. En este sentido, el nivel léxico ocupa un papel protagónico, ya que es lo primero que los hablantes identifican como perteneciente a un estrato social, género, región, etc.

Los estudios de corte sociolingüístico han dado cuenta de cómo se ordena la variación a partir de la producción de datos de los hablantes. Parte integral de los estudios de variación ha sido la exploración de las creencias y actitudes sobre el lenguaje. Los estudios de dialectología perceptual $^{3}$, que centran su atención en la delimitación que ofrecen los propios hablantes sobre su lengua y de sus mismas variedades, tienen su anclaje en la lingüística folk, la cual explora las creencias y actitudes conscientes ante los hechos lingüísticos. Sin embargo, tales estudios son casi nulos, por mencionar algunos, se encuentra el trabajo de Serrano (2002), y el relativamente reciente trabajo de Erdösova (2011) entre estudiantes de la Universidad Autónoma del Estado ${ }^{4}$ : En dicho trabajo se analiza la percepción que tienen los hablantes de diferentes variedades del español; basándose en una metodología sociolingüística, al analizar las actitudes que tienen los hablantes, y mediante la dialectología perceptual al analizar la consciencia que tiene estos tienen acerca de las variación dialectal del español. Es interesante señalar las coincidencias que existen entre el trabajo de Erdösova y Serrano, por mencionar algunas, ambos autores encuentran que el habla norteña es la que más fácilmente identifican los hablantes; esto quizás se deba a lo estereotipada de la misma. Curiosamente, es también percibida por los hablantes como la más incorrecta. En el trabajo de Serrano (2002), se concluye que los hablantes segmentan el español del país a través de topónimos y estereotipos sociolingüísticos diversos (como "hablar fuerte/ cantadito", "comerse los sonidos",, etc.). De esta forma, el estudio de la percepción de las diferencias dialectales hace asequibles los conceptos folk que se tienen sobre la diversidad en la lengua. En la variante peninsular, Moreno Fernández \& Moreno Fernández (2004) analizan algunos aspectos de dialectología perceptual española y cómo estos se relacionan con la situación lingüística de España. Otro estudio que versa sobre el mismo tema es el de Quesada Pacheco (1996), en el cual, el autor intenta trazar una división dialectal del español de América con base en la opinión de sus hablantes. En tal estudio, se busca que los hablantes evalúen las similitudes y diferencias acerca de la forma de hablar de los entrevistados de otro país. Asimismo, Quesada Pacheco recurre a plasmar en mapas los resultados obtenidos, algo que coincide con Serrano (2002) y con Serrano \& Morúa (2004) con la finalidad de mostrar la división dialectal hispanoamericana de acuerdo con la percepción de dichos hablantes.

En este trabajo se abordan las concepciones que los hablantes de español de las poblaciones costeras sonorenses estudiadas tienen sobre tres palabras particulares, los resultados se detallarán en los siguientes apartados.

\section{Variación léxica y regionalismos}

El léxico experimenta variación diacrónica, caracterizada por la desaparición de palabras arcaicas, y por la aparición de otras (neologismos). También experimenta variación diatópica, es decir, aquélla que tiene que ver con la región (se profundizará en el concepto más adelante). Existe también la variación de estrato $^{6}$, esta tiene que ver con las diferencias encontradas entre diferentes grupos sociales. Por último, el léxico experimenta variación a nivel diafásico, este tipo de variación tiene que ver con el registro (ámbito por excelencia de la Pragmática).

La variación léxica es observable, en los sustantivos. Por ejemplo, Everardo Mendoza (2002) señala que chuparrosa se utiliza más en Sinaloa, mientras que en el centro se utiliza más colibrí; en cambio, cochi ('cerdo') es una forma privativa de Sinaloa y del sur de Sonora, pero no es utilizada en el resto del país, que prefiere formas como puerco. A este tipo de variación se le conoce como regionalismo. En este sentido, Rona (1969:137) define regionalismo ${ }^{7}$ como 
"todo elemento privativo de una región"; señala que si se considera el origen de la palabras, entonces se trata de un regionalismo diacrónico. Añade, además, que existen los mexicanismos sincrónicos, vocablos que pueden proceder o no de las lenguas prehispánicas, los cuales son conocidos por la mayoría de los mexicanos pero ausentes en los idiolectos no mexicanos; existen también los mexicanismos diacrónicos, es decir, aquellas palabras que tuvieron origen en territorio mexicano y que, por lo general, provienen de alguna lengua indígena razón por la cual, están expresados como indigenismos mexicanos.

Dentro de los indigenismos es posible distinguir los de uso exclusivo en territorio nacional y aquellos cuya extensión geográfica rebasa las fronteras y que se usan en el español en general. Así por ejemplo, bato es definido por la RAE (2001) mediante dos entradas distintas $^{8}$. La primera define bato como hombre tonto rústico o de pocos modales. La segunda entrada de bato es "padre". Sobarzo, en su vocabulario sonorense, define bato, de una forma que contrasta con los hallazgos que se presentan en este trabajo. Por un lado, atribuye a bato, un origen griego. Por otro lado, difiere en la edad. Como se mostrará en los siguientes sub-apartados, bato alude a un hombre de 30 años. No obstante, Sobarzo (2006:34) lo define como una persona joven. Veamos, pues, la definición de este:

\footnotetext{
Bato. S.m. fam. Muchacho, chico, jovenzuelo. En su sentido propio significa hombre tonto o rústico y de pocos alcances, derivado del griego battos, tartamudo, substantivo que a su vez se derivó de Battos, rey de Cirene, famoso por su tartamudez. Nuestra expresión alude, con sentido afectivo a la inexperiencia y poco aviso de juventud.
}

Por otra parte, Sobarzo define morro, más por la prominencia de sus rasgos fónicos que por un significado específico, que para este autor es de burla:

Morro. m. Burla, mofa. Alude al gesto que se produce al abuchear con murmullos, repitiendo la exclamación joooh!, joooh!, ¡uuuh!, ¡uuuh!, ¡uuuh! Se prolongan los labios redondeándolos para pronunciar la $o$ y más aún la $u$, todo lo cual se acentúa si se sostiene la emisión de la voz. Entonces, el gesto se caracteriza por el morro saliente que forman los labios abultados. Le hizo morro por le hizo burla. (2006:158)

Finalmente, plebe, se define como los integrantes más pequeños de la sociedad o de una familia. Para tal palabra, no fue posible encontrar definición alguna por parte de Sobarzo. Ninguna de estas acepciones concuerda con las utilizadas en Bahía de Kino y Guaymas, Sonora; por lo tanto, al tener un significado de uso exclusivo de estas poblaciones sonorenses (y de algunas otras en el Estado), pueden considerarse regionalismos propiamente.

\section{Metodología}

Como se comentó en la introducción, el estudio se realizó con la colaboración de 24 personas en total entrevistadas en Bahía de Kino, Guaymas. El rango de edades fue, en la medida de lo posible, simétrico, de manera tal que para un hablante masculino de 48 años, se buscó a una mujer cuya edad fuera similar. Respecto a la cuantificación, se optó por utilizar la moda como herramienta estadística, ya que esta es útil al momento de dar cuenta del elemento que ocurre con mayor frecuencia cuando no se cuenta con un gran número de datos; la moda da cuenta del valor más común en una muestra. Se entrevistó a 24 hablantes en total: 12 de Bahía de Kino y 12 de Guaymas, seis mujeres, y seis hombres en cada puerto estratificados por edad, sexo y grado de instrucción.

Para investigar las variantes léxicas se les preguntaba a las personas: ¿a qué le llamas plebe?, ¿a qué le llamas morro?, ¿a qué le llamas bato? De este modo, las personas proporcionaron una definición de cada uno de los sustantivos. Finalmente, se utilizó un criterio formal, que consistió en preguntar a los hablantes por la palabra en sí: los diminutivos, la forma plural, así como indagar si se podía usar el femenino para cada palabra, es decir, se formularon preguntas como "ise puede decir 'bata'?”,"'Se puede decir 'batito'?”, “Se puede decir 'morra'?", ¿Se puede decir 'morrita', 
'morrito'?" De igual forma, se optó por la observación directa, tratando de cruzar estas observaciones con las características sociales de los informantes (sexo, región, edad y ocupación). A continuación, se hará un esbozo de cada una de ellas.

\section{El bato 9}

La palabra bato es frecuente en la región del Noroeste. Sin embargo, los estudios referentes a su uso y origen son inexistentes. Ninguna de las personas entrevistadas identificó su origen.

De 24 informantes, solo dos, un hombre y una mujer $(8.3 \%)$, negaron el uso propio de la palabra; es decir, enfatizaron que la usan los demás, no ellos. Al parecer, la variable sexo, independientemente de esto, no presenta mayor cambio: hombres y mujeres no hacen distinción alguna respecto a la palabra.

En cuanto a la región y la percepción de los hablantes, definitivamente hay un cambio. Mientras que en Guaymas no tiene carga peyorativa, entre los habitantes de Bahía de Kino sucede lo contrario. En Guaymas, solo un hombre $(4.6 \%)$ de nivel alto refirió que le disgustaba usar esta palabra, ya que refiere al habla de "los cholos", por lo demás, el 95.8\% de la población aseveró que la palabra bato no es peyorativa:

"No es peyorativa, es como decir "este muchacho". Oye, bato, ¿me ayudas a cargar algo?”. Es una forma de decir 'muchacho', pues" (JRL, hombre, grado de instrucción alto, 41 años, residente de Guaymas.)
Al parecer, la gente utiliza bato para referirse a un hombre cuya edad mínima oscila alrededor de los 30 años; la moda obtenida para este rango de edad fue de 8 . El $8 \%$ de la población señaló que bato refiere a una persona cuya edad es mayor o igual a los 15 años. El 24\% indicó que bato alude a un hombre de 20 años en adelante. Por otra parte, en Bahía de Kino bato es percibida como una palabra peyorativa. Nueve hablantes (el 75\%) opinaron que es una palabra de uso despectivo:

Lo usamos, pero es más bien una palabra peyorativa de decir "este bato". O sea, más bien como un tono de rechazo... bato. Aquí empleamos la palabra cuando no estamos de acuerdo con esa persona. $\mathrm{O}$ sea, aquí la palabra bato no se le dice a cualquiera, se le dice a quien se la gane (MT, hombre, grado de instrucción bajo, 48 años, residente de Bahía de Kino).

A un amigo que no lleva una vida bien. Te refieres a alguien que... yo sugiero que deshonesto. Sí, es peyorativo, es como... En la vida hay tres clases: muy bajo, bajo y muy alto; bato es porque no lo quieres ni mencionar. Es una persona deshonesta que nomás está esperando que te des la vuelta y robarte algo. Ese es un bato. (FJ, hombre, grado de instrucción medio, 35 años, residente de Bahía de Kino).

Es un hombre: "este bato" yo oigo decir a los demás. Normalmente esas palabras las crearon los drogadictos: que el bato, que la morra... Normalmente es una palabra de las banditas (MB, mujer, grado de instrucción alto, 44 años, residente de Bahía de Kino).

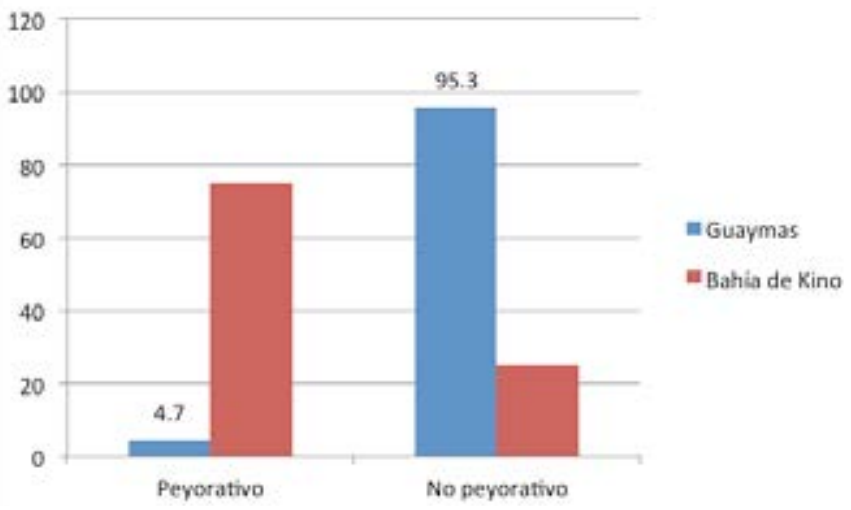

FIGURA 1. Uso peyorativo de la palabra bato 
En Bahía de Kino, las personas no identificaron un rango específico de edad para esta palabra, por esta razón fue difícil determinar la moda. En lo que concierne al grado de instrucción, no parece haber diferencias entre esta variable y la percepción de la edad. Aunque se pudo observar que la gente de mayor grado de instrucción proporcionó por sí misma el origen de la palabra. De igual forma, dicho grupo identificó que bato era una palabra regional, tanto de Guaymas como de Bahía de Kino, pero que en poblaciones sureñas como Sinaloa ${ }^{10}$ y Agua Prieta ${ }^{11}$, es más común decir otras palabras.

Finalmente, respecto a los criterios formales (diminutivo y femenino) es interesante resaltar algunas cuestiones. Dos personas $(8.3 \%)$ consideraron que probablemente se podría decir "batito". No obstante, aclararon que no era muy común y que era mejor decir "el morrito". El resultado indica que el uso del diminutivo no está plenamente aceptado entre los hablantes. Respecto al uso femenino del sustantivo, el resultado es contundente: el $100 \%$ de los hablantes negó el uso de "bata":

Bata no se escucha bien (ACE, hombre, 27 años, grado de instrucción medio, residente de Guaymas).

No, (risas) no se puede decir bata (PA, mujer, 21 años, grado de instrucción alto, residente de Guaymas).

No, ese nomás se le dice a un hombre. No le dicen a ella (JF, hombre, 17 años, grado de instrucción medio, residente de Bahía de Kino).

A manera de conclusión, se puede afirmar que bato refiere a un hombre cuya edad oscila entre 25 a 38 años. En Guaymas la acepción de tal vocativo es neutra, si cabe el término; las personas lo utilizan sin una carga peyorativa. En Bahía de Kino, en cambio, es peyorativo, incluso innombrable, por lo que es posible afirmar que los hablantes de este último lugar perciben el uso de tal palabra como parte del habla marginal del puerto.

Es necesario acentuar el rechazo rotundo en cuanto al uso de la palabra bata, con esto es posible afirmar que el vocativo bato designa exclusivamente a un hombre. Aunque no hay unanimidad entre los hablantes acerca de por qué no es posible decir bata. Resultaría interesante para futuros estudios rastrear el origen histórico de la palabra e indagar si existe una forma similar femenina.

\section{El morro}

La palabra morro es frecuente en el habla de los puertos sonorenses estudiados, y en todo el estado de Sonora. Sin embargo, su uso queda restringido a ciertos sectores de la población ${ }^{12}$. Al menos esto fue lo que pude comprobar a lo largo de las entrevistas. Morro fue el sustantivo que los hablantes más fácilmente identificaron por sí mismos, sin que se forzaran sus respuestas; todos coincidieron en que proviene "de los cholos"13.

La variable sexo ${ }^{14}$ no presenta diferencias significativas. Hombres y mujeres perciben de forma similar el uso, origen y variaciones de la palabra, por lo que se deduce que su uso no presenta diferencias significativas entre ambos puertos. Tal vez esto se deba a que esta palabra puede flexionarse en femenino, es decir, morra, sin que haya objeción alguna por parte de los hablantes.

Las variedades percibidas en ambas poblaciones no parecen contrastar mucho. El 95\% de los hablantes de Bahía de Kino ${ }^{15}$, y el $80 \%$ de los habitantes de Guaymas identifican que esta palabra proviene de los cholos:

Es una palabra más vulgar que no cualquiera la
usamos. Morro es como de cierto tipo de sociedad.
Cuando era chico, yo busqué en el diccionario y
resulta que morro es un montículo de tierra, enton-
ces yo dije "no" (JN, hombre, 44 años, grado de
instrucción alto, residente de Guaymas).

El prestigio del cual goza esta variable es escaso y está asociado al grado de instrucción. De nuevo, los hablantes con mayor grado de instrucción (54\%) identifican la palabra como parte del léxico de los cholos; de estos, la mitad señala que se trata de una palabra fuerte y que nunca la utilizarían. Un solo hablante propuso como alternativa para morro y esta fue lepes. 
Él mismo comentó que lepes no era una palabra oriunda de Guaymas, más bien de Agua Prieta y de Sinaloa, pero dijo que prefería usar esta en vez de morro.

Finalmente, el rango de edad que designa morro, abarca desde 1 año hasta $19^{16}$. Al ser considerada, esta palabra como vulgar, los hablantes señalaron que prefieren utilizar muchacho/muchacha, (22\%), niño o niña (15\%). El rango de edad parece no tener importancia, aunque los hablantes tienen claro que refiere a una persona joven. La edad percibida parece cambiar en los grupos de grado de instrucción: en el grupo de mayor grado de instrucción, el promedio es de 20.5 años; mientras que en el grupo de menor grado de instrucción, cambia a 18.5. Al parecer morro, en el nivel de estudios altos, abarca un mayor rango de edad. En el grupo de estudios medio y bajo, se restringió la edad hasta 17 años. Un interlocutor dijo que morro refería a alguien grande, ya de $20^{17}$.

Respecto a los criterios formales, las flexiones de diminutivo y femenino no presentan mayor variación; como se esperaba, es una palabra más versátil que admite ambas flexiones. Así, resulta admisible decir: morrito(s), morrita(s). Una sola persona de nivel de instrucción alto restringió el uso de morro a "hombres adolescentes". A grandes rasgos, es posible concluir que la edad promedio de un morro es de 16.9 años; abarca desde los 11 años hasta los 29 .

No deja de llamar la atención el hecho de que en ambos puertos se muestre un comportamiento morfológico y semántico distinto entre las palabras bato y morro, sobre este último, es posible afirmar que equivale a la palabra chavo del centro del país. Bato, definitivamente, no es utilizado en el centro del país, aunque algunas personas lo identifican como una palabra de las hablas norteñas.

\section{Los plebes}

Esta palabra es, definitivamente, la más característica de Sonora y Sinaloa, y tal vez la más susceptible a malinterpretaciones por parte de un hablante foráneo. Sin embargo, plebes, en las regiones en las que se realizó el trabajo de campo, refiere a un grupo de niños, incluso es utilizado con cierta carga afectiva por parte de los adultos para dirigirse a un grupo de niños y adolescentes.

En cuanto a la variable sexo, el análisis muestra que tanto hombres como mujeres usan plebes para referirse a los niños en general, sin distinción de edad, tal vez se deba a que es un vocativo regional muy extendido en todos los grupos sociales que carece de carga peyorativa alguna.

Respecto a la región, no parece haber cambio alguno en cuanto la percepción de la misma o más aún, en cuanto al rango de edad. La edad promedio a la que refiere la palabra plebes es de 15.7 años en Guaymas y de 14.3 en Bahía de Kino. El grado de edad que designa plebes abarca desde un año hasta los 19 años, aproximadamente. A simple vista, puede parecer similar a morro, pero, más bien, la diferencia entre ambos estriba en que el primero abarca edades más cortas, mientras que el segundo se aplica a edades más amplias, rebasando incluso la adolescencia. En otras palabras, un plebe no se le puede decir a un adulto joven, mientras que morro sí.

La variable grado de instrucción no parece mostrar resultados contrastantes. Tanto las personas pertenecientes al grupo de mayor grado de instrucción, como las pertenecientes al nivel medio perciben que plebes refiere a un grupo de edad menor. El $92 \%$ de los habitantes de Guaymas definió plebes mediantes las frases "el más chiquito" "el niño", "un niño". El 8\% restante señaló que puede utilizarse para jóvenes de 20 años o más; sin embargo, aclararon que tal uso se utilizaba dentro de un contexto de juego.

Pues yo les digo a mis amigas, “joigan, plebes! ¿Qué vamos a hacer hoy en la noche?” (BES, mujer, 27 años, grado de instrucción medio, residente de Guaymas).

\footnotetext{
Al más chiquito de la casa (JR, hombre, 44 años, grado de instrucción alto, residente de Guaymas).

A los niños, y pues yo que tengo veintitrés y le digo plebe a mi hermano que tiene dieciocho
} 
(AF, mujer, 23 años, nivel de estudios medio, residente de Bahía de Kino).

Yo, a los míos, a mis chamacos, les digo plebes: “¡buenas noches, plebes! --¡buenas noches, amá! [mamá]" (MB, mujer, 44 años, grado de instrucción medio, habitante de Bahía de Kino).

Respecto a los criterios formales, el $72 \%$ señaló que es un sustantivo plural, principalmente, aunque también puede usarse para vocativo singular: “¡ey, plebe!”. El 92\% negó el uso del diminutivo, así como del femenino .

Las palabras plebe, bato y morro son palabras regionales de las poblaciones de Guaymas y Bahía de Kino que forman parte de la identidad lingüística local; pude observar que en muchas respuestas predominó el comentario "son palabras de aquí, de la región".

\section{Conclusiones}

En este trabajo se estudió la percepción, creencias, actitudes e intuición que sobre algunas palabras regionales tienen los hablantes entrevistados en Bahía de Kino y Guaymas. De esta manera, el presente estudio centró su atención en analizar lo que ellos proponen, más que en documentar definiciones de diccionario, que como se planteó en páginas anteriores, no siempre dan cuenta de la diversidad semántica de las palabras caracterizadas como regionalismos.

De este modo, de acuerdo con lo expresado por las personas, bato refiere tanto en Guaymas como en Bahía de Kino a un individuo masculino de aproximadamente 30 años. El principal hallazgo fue la disparidad respecto a las virtudes que podría tener una persona a quién se le dice bato. En otras palabras, mientras que en Bahía de Kino, el 75\% de las personas afirmaron que es una palabra despectiva y que refiere a alguien, prácticamente innombrable y deshonesto, en Guaymas, al parecer no tiene una carga semántica peyorativa, al menos el 95.3\% de los hablantes afirmó que su uso no es peyorativo. Se desconoce a qué se debe esta diferencia de registro y de estrato, por lo que resultaría muy interesante explorar esta palabra en futuras investigaciones, mediante una prueba de actitudes lingüísticas. Dada la variación que presenta la palabra bato respecto a cómo es percibida en los diferentes puertos, sería también conveniente hacer un estudio en tiempo real y tiempo aparente para poder determinar cuándo empezó a cambiar la palabra, y cómo la acepción de esta cambió en ambos puertos. La conclusión en la que sí existió acuerdo general entre los hablantes de ambos puertos es que no se puede decir "bata".

La palabra morro presenta un uso más o menos aceptado entre los hablantes, con la previa aclaración de que dicha palabra proviene del habla de los cholos. Nuevamente, nos encontraríamos, muy posiblemente ante una cuestión de actitudes. Por este motivo, sería importante explorar esta palabra, bajo dicha línea de estudio, ya que seguramente, el análisis arrojará resultados interesantes. Respecto a la variable región, no parece existir diferencia entre de uso entre ambos puertos, salvo la variable, edad, que como ya se explicó anteriormente, muy posiblemente tendría que ver con el grado de marginalidad de los puertos. Por lo demás, la percepción de los hablantes, parece ser homogénea en todas las variables lingüísticas y sociales que se estudiaron.

Finalmente, en cuanto a la palabra plebes se puede afirmar que se trata de un regionalismo privativo de los estados de Sonora y Sinaloa, $\mathrm{y}$, tal vez, por esta razón sea la más uniforme entre ambos puertos y la que menos variación presentó. Su registro es amplio, y puede ser utilizado en tono de broma entre adultos. Con base en las respuestas que dieron los hablantes, se trata de un sustantivo plural para niños muy pequeños y que puede hacer referencia incluso a adolescentes. Por lo que se observó, y por lo que los mismos hablantes expresaron, es una palabra que usan mucho las madres, principalmente, para dirigirse a sus hijos.

Al analizar las palabras morro, plebe y bato se concluye, sobre todo respecto a esta última, que nos encontramos ante cierta variación e inestabilidad en cuanto a su origen, significado y percepción. En cambio, morro y 
plebe presentan un uso más bien estable en ambos puertos. Finalmente, conviene precisar que de las tres palabras estudiadas, plebe, es privativa de los estados de Sonora y Sinaloa, mientras que bato y morro, parecen ser de uso general en el Noroeste Mexicano (Baja California, Sonora y Chihuahua).

\section{Notas}

1. La fotografía fue tomada por la autora del artículo.

2. Sin embargo, tales juicios pasan por un proceso de concientización por parte del hablante.

3. Algunos autores, como Erdösová (2011) utilizan el término "Dialectología Perceptiva". En este trabajo, se utilizará el término "Dialectología Perceptual".

4. Perteneciente al estado de Toluca.

5. Es decir, la elisión y aspiración de /s/ en posición implosiva.

6. La variable estrato es una de las que más controversias ha suscitado, ya que no hay acuerdo general respecto a qué factores son los que hacen que una persona sea considerada como de estrato bajo, alto o medio. La aplicación de esta variable, indudablemente tiene que ver con la Sociología.

7. Respecto a los regionalismos, estos forman parte de la variación léxica, y son los que más fácilmente perciben los hablantes (junto con los regionalismos fónicos) una vez que viajan de región a región. Ambrosio Rabanales (1953) profundiza al respecto y establece que existen dos tipos de regionalismos. El primer tipo es el diatópico, y es aquel cuyo resultado obedece a la comparación dialectológica de diferentes regiones, en aras de encontrar un elemento distintivo, o en nuestra terminología, un elemento privativo de cada una de ellas. El segundo tipo de regionalismo es el sintópico, el cual no es más que el resultado de una sola región estudiada.

8. No hay consenso entre los hablantes para escribir esta palabra. Algunos prefieren escribir bato, mientras que otros más optan por vato. Yo misma he constatado que en los puertos estudiados la mayoría de las personas prefieren escribir bato. Sobarzo (2006:37) opta por escribir bato, en vez de vato.
9. Se prefirió usar el determinante "el" ya que este es una característica de todo el Noroeste para determinar o cosificar, por así decirlo, a las personas. Además, es el contexto bajo en el cual aparece la palabra. De aquí en adelante, se hará lo mismo con las demás palabras.

10. Estado ubicado al sur del estado de Sonora. Curiosamente, los sonorenses consideran que toda población y persona que provenga de lugares que se residan fuera de la Ciudad de Hermosillo, ya no son sonorenses, por lo tanto, a tales personas se les dice wachos.

11. Población perteneciente al estado de Sonora, limítrofe con Estados Unidos.

12. Específicamente a los cholos. Más adelante será discutido.

13. Un cholo en la región estudiada es identificado normalmente como un hombre que se viste de forma exageradamente holgada, que es drogadicto y en muchos de los casos se dedica a delinquir.

14. Solo un hombre relacionó esta palabra con un rasgo sexual. Sin embargo, esto se debe a una característica personal del hablante, y no a un rasgo que se relacione con la palabra morra.

15. En Bahía de Kino, una mujer de 44 años, perteneciente al grupo de alto grado de instrucción, comentó que prefería usar buki, en vez de morro.

16. Respecto a la edad, no es muy clara la frontera entre morros y plebes. Para definir morro, los hablantes recurrían a la definición de plebes. Una posible explicación al respecto es que el rasgo distintivo que divide ambas palabras es que uno, morro, tiene poca aceptación entre los hablantes.

17. En este sentido, el hecho de que los hablantes mencionen que morro refiere a alguien ya grande, ya de 20 , se deba, probablemente, al nivel de vida. Se ha dicho que la juventud es un lujo de los países desarrollados, ya que en tales lugares, por poner un ejemplo, se prolonga el inicio de la vida sexual, no hay embarazos no deseados ni precoces. Así, hombres y mujeres deciden formar una familia después de los 35 años; en lugares con rezago educativo y con pobreza, como Bahía de Kino y Guaymas, ocurre lo contrario: a los 35 
años, los hombres y mujeres ya son abuelos, o están a punto de serlo (por lo que pude constatar con varios hablantes). De esta forma, resulta lógico que conciban a un joven de 20 años, como alguien ya grande.

\section{Referencias}

Álvarez González, Albert. 2006. La variación lingüistica y el léxico: Conceptos fundamentalesyproblemas metodológicos. México: Universidad de Sonora.

Bloomfield, Leonard. 1933 [1961]. Language. Nueva York: Holt, Rinehart \& Winston.

Erdösová, Zuzana. 2011. El español de México en los ojos de sus hablantes. Un estudio desde la sociolingüística y la dialectología Perceptiva. Lengua y Voz, Año 1 (1): 57-81.

Gould, Peter \& Rodney White. 1972. Mental Maps. Harmondsworth, UK: Penguin.

Mendoza Guerrero, Everardo. 2002. El léxico de Sinaloa. México, D.F.: Siglo XXI /El Colegio de Sinaloa.

Moreno Fernández, Francisco \& Moreno Fernández Juliana. 2004. "Percepción de las variedades lingüísticas de España por parte de hablantes de Madrid". Lingüística española actual XVI (1): 5-38.
Morúa Leyva, María del Carmen \& Serrano Morales Julio César. 2004. "2000 kilómetros de por medio. Dialectología perceptual contrastiva del español mexicano". En: Memorias del VII Encuentro Internacional de Lingüística en el Noroeste, tomo 2, María del Carmen Morúa Leyva \& Rosa María Ortíz Ciscomani (eds.). Hermosillo: Universidad de Sonora: 253-276.

Niedzielski, N.A.\& Dennis R. Preston. 2003. Folk Linguistics. Berlín-Nueva York: Mouton de Gruyter.

Real Academia Española. 2001. Diccionario de la lengua española. 22a ed. Madrid: España.

Preston, Dennis R. 1989. Perceptual Dialectology: Nonlinguists' views of areal linguistics (Topics in Sociolinguistics, 7). Dordrecht, Holland, and Providence, RI: Foris.

Quesada Pacheco, Miguel Ángel. 1996. El español de América Central. En Manuel Alvar (dir.) Manual de dialectología hispánica: El español de América. Barcelona: Ariel. pp. $101-115$

Rabanales Ambrosio. 1953. Introducción al estudio del español de Chile. Determinación del concepto de chilenismo. Santiago de Chile: Editorial Universitaria. 
Rona, J. P. 1969. ¿Qué es un americanismo? El Simposio de México. Actas, informes y comunicaciones del Programa Interamericano de Lingüística y Enseñanza de Idiomas. México: UNAM. pp. 149-190.

Serrano Morales, Julio César. 2002. ¿Cuántos dialectos del español existen en México?
Un ensayo de dialectología perceptual. http://unam.academia.edu/JulioSerrano. Mayo 2017.

Sobarzo, Horacio. 2006. Vocabulario sonorense. Hermosillo: Programa Editorial de Sonora.

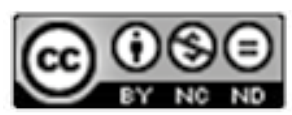

Este obra está bajo una licencia de Creative Commons Reconocimiento-NoComercial-SinObraDerivada 4.0 Internacional. 